\title{
LA REALIDAD DE LOS PUEBLOS \\ INDÍGENAS EN CHILE: \\ UNA APROXIMACIÓN SOCIODEMOGRÁFICA PARA CONTRIBUIR AL DISEÑO DE POLÍTICAS PÚBLICAS PERTINENTES
}

Verónica Figueroa Huencho 


\section{VERÓNICA FIGUEROA HUENCHO}

Doctora en Management Sciences (ESADE), administradora pública (Universidad de Chile). Profesora asistente del Instituto de Asuntos Públicos, Universidad de Chile. Colaboradora académica del Instituto de Dirección y Gestión Pública (IDGP) de ESADE. Ha dictado clases para el MBA de ESADE y para el Programa de Formación de Líderes Indígenas de la Universidad de Deusto. Ha trabajado como consultora para la Fundación Forum Universal de las Culturas (Barcelona), para el Banco Interamericano de Desarrollo (Washington) y para la Organización de Estados Iberoamericanos para la Educación, la Ciencia y la Cultura (OEI). Sus líneas de investigación son políticas públicas, gestión pública, multiculturalismo y gestión pública intercultural. 


\section{LA REALIDAD DE LOS PUEBLOS INDÍGENAS EN CHILE: UNA APROXIMACIÓN SOCIODEMOGRÁFICA PARA CONTRIBUIR AL DISEÑO DE POLÍTICAS PÚBLICAS PERTINENTES}

\section{INTRODUCCIÓN}

Durante el último tiempo se ha prestado enorme importancia al fenómeno multicultural que se está produciendo en la mayoría de las sociedades, especialmente por los efectos que produce en ámbitos como el político, el económico o el social, y las necesidades de acción que impone a los diferentes actores al interior de estas sociedades, como los Estados, las empresas o la ciudadanía.

La multiculturalidad se refiere a la existencia en el espacio social de culturas e identidades diversas. Esta diversidad se da en las sociedades actuales y es posible que se acreciente en las futuras, como consecuencia de los procesos de globalización, de la revitalización de movimientos de minorías nacionales, así como de los procesos de movilidad de poblaciones a partir del fenómeno de la inmigración (Vallescar Palanca, 2000).

En ese sentido, la realidad multicultural actual implica la búsqueda de nuevas formas de convivencia armónica y respetuosa. Como señala Alan Touraine, "el multiculturalismo sólo tiene sentido si se define como la combinación, en un territorio dado, de una unidad social y de una pluralidad cultural mediante intercambios". Agrega que "es necesario articular lo particular y lo universal porque sólo así es posible la convivencia" (Touraine, 1995).

En términos de multiculturalidad, América Latina posee una diversidad que se manifiesta en la existencia de más de 400 pueblos indígenas, cada uno de los cuales tiene su idioma, su organización social, su cosmovisión, su sistema económico y su modelo de producción adaptado a su ecosistema (Deruyttere, 1999).

Sin embargo, la mayoría de estos pueblos han sufrido un proceso de "asimilación" histórica con la cultura dominante, que los ha obligado en muchos casos a dejar de lado su propia cultura e identidad, situación que se ha agravado con los crecientes procesos migratorios hacia zonas urbanas y los procesos de modernización económica y cultural (Ancán, 1994).

Es por ello que algunos autores señalan que el multiculturalismo en Latinoamérica no es un fenómeno problemático reciente en términos de convivencia y entendimiento, sino que lleva más de 500 años de existencia (Domenech, 2003). Como señala Martín Hopenhayn, entre otros, el fenómeno está ligado históricamente al período de colonización y conquista, cuando las relaciones sociales se basaban en la "dialéctica de la negación del otro" (Hopenyan, 2001).

Podríamos decir que en el contexto latinoamericano se presenta un gran desafío: atender las demandas de los pueblos indígenas evitando los conflictos entre estos y las sociedades "dominantes", pero sin perder la perspectiva multicultural, sin dejar de lado aspectos culturales, valóricos y normativos. Solo así las relaciones podrán ser justas y 
beneficiosas para ambos (Kymlicka, 2003). Junto con lo anterior, aparece otra variable que impone acciones específicas a los gobiernos con respecto al desarrollo de estos pueblos: la correlación entre etnicidad y pobreza (Plant, 1998).

Chile no constituye una excepción. Actualmente existen nueve pueblos indígenas que componen más del 4,6\% de la población mayor de 14 años. Sin embargo, al igual que el resto de Latinoamérica, la mayoría de ellos están asociados a condiciones de pobreza y marginalidad de carácter alarmante, lo que ha derivado en una pérdida progresiva de su bagaje cultural.

En este artículo nos abocamos a la tarea de identificar y analizar aquellos datos que nos permitan tomar una "fotografía" de los pueblos indígenas en Chile en la actualidad a partir de una serie de datos cuantitativos que diagnostiquen su situación sociodemográfica y cultural. Para este análisis realizaremos una comparación entre los datos disponibles a partir del Censo de 1992 (en el que se incluyó por primera vez una pregunta relacionada con la temática indígena), el último Censo de 2002, la encuesta de caracterización socioeconómica CASEN 2006 y 2009, así como datos de la CONADI y el Instituto de Estudios Indígenas (IEI) de la Universidad de la Frontera. Toda esta información nos permitirá comprender de forma acabada algunas transformaciones sufridas por estos pueblos, y entregar, así, algunas reflexiones y recomendaciones a la formulación de políticas públicas indígenas pertinentes.

\section{LA ESPECIAL SITUACIÓN DE LOS PUEBLOS INDÍGENAS LATINOAMERICANOS}

Son varias las situaciones complicadas que deben enfrentar los pueblos indígenas. Si bien, como hemos planteado anteriormente, la realidad latinoamericana está marcada por la diversidad y la multiculturalidad, esta diversidad no ha implicado, por lo general, respeto ni un mayor nivel de pluralismo o integración cultural. La multiculturalidad tiene una expresión especial en América Latina, marcada por una historia colonial que ha creado una división entre pueblos indígenas-resto de la sociedad que es específica. Allí encontramos a los pueblos originarios que se encuentran con graves dificultades para conseguir que su cultura sobreviva (Etxeberria, 2004).

Por una parte, esta negación de la heterogeneidad cultural que ha caracterizado el nacimiento de la mayoría de los Estados nacionales se ha perpetuado en el tiempo mediante sistemas de reproducción social y cultural (Hopenhayn y Bello, 2001). Han existido diversas formas mediante las cuales los pueblos indígenas se han incorporado a las comunidades políticas: desde la conquista y la colonización de sociedades que anteriormente gozaban de autogobierno hasta la inmigración voluntaria de individuos y familias (Kymlicka, 2003).

Tal como señala Kymlicka, por regla general los pueblos indígenas han estado aislados del proceso de formación estatal y de aquellos que afectaban su propio desarrollo (Kymlicka, 2003). Este proceso ha significado para muchos de los indígenas la pérdida progresiva de tierras, el quiebre de economías comunitarias, la pérdida de derechos y representatividad y la marginación de procesos políticos, entre otros. 
De esta forma, en términos de convivencia, las relaciones entre los pueblos indígenas y las sociedades mayoritarias han estado marcadas por la existencia de conflictos y por una realidad de discriminación que ha perdurado a través de los años (Bello y Rangel, 2000) y que ha venido marcada por las "reivindicaciones de las culturas oprimidas y por su lucha contra la hegemonía de una cultura que pretende identificarse con lo universal" (Vallescar Palanca, 2000).

Por otra parte, como resultado de procesos históricos, otro elemento que ha caracterizado la realidad de estos pueblos ha sido la pobreza y la marginación. En este sentido, la negativa situación socioeconómica de estos pueblos se ha visto agravada por políticas sistemáticas que no han tomado en cuenta sus necesidades reales y por acciones emprendidas sin "identidad" (Valdés, 2000). La mayoría de estos pueblos han sido incorporados de modo marginal y precario a los proyectos nacionales (Máiz, 2004), dándose así una combinación de injusticia cultural y material.

De acuerdo con varios estudios, la mayoría de los indígenas son pobres. De la población latinoamericana que vive en la extrema pobreza un cuarto son indígenas (Pirttijärvi, 1999). Los pueblos indígenas representan el 10\% de la población de la región y sus niveles de ingreso, al igual que sus indicadores de desarrollo humano como la educación y las condiciones de salud, han quedado sistemáticamente a la zaga en relación con los del resto de la población (Hall y Patrinos, 2004).

En este sentido, los datos son claros. De acuerdo con el estudio realizado por Hall y Patrinos (2004), ser indígena aumenta las probabilidades de un individuo de ser pobre. Controlando los factores básicos que están sabidamente asociados con la pobreza, tales como la edad, la educación, la situación laboral y la región dentro de un país, ser de origen indígena aun aumenta de manera más significativa las probabilidades que un individuo tiene de ser pobre.

Tabla 1

AUMENTO PORCENTUAL DE LA POSIBILIDAD DE SER POBRE SI SE ES INDÍGENA

\begin{tabular}{|l|c|c|}
\hline País & Comienzos de 1990 & Último año disponible \\
\hline Bolivia & 16 & 13 \\
\hline Ecuador & -- & 16 \\
\hline Guatemala & 11 & 14 \\
\hline México & 25 & 30 \\
\hline Perú & -- & 11 \\
\hline
\end{tabular}

Fuente: Hall y Patrinos, 2004.

Además de la discriminación y el racismo, las principales causas de esta situación son atribuidas a las reformas liberales del siglo XIX, que al introducir la noción de propiedad privada de las tierras generaron una pérdida progresiva de tierras y un quiebre de las economías comunitarias. La globalización, la modernización, los ajustes económicos, etc., han producido reestructuraciones tecnológicas, políticas y socio-culturales que han agudizado la precaria situación de la mayoría de los pueblos indígenas (Hernández 
y Calcagno, 2003). A estos procesos se sumaron, como agravantes, la migración campociudad y la inserción laboral (Plant, 1998).

Efectivamente, son estos pueblos los que presentan los peores indicadores económicos y sociales y son, en gran medida, los más pobres de la región (Hall y Patrinos, 2004). En los escasos estudios comparativos existentes, las cifras de pobreza desagregadas por origen étnico-racial muestran diferencias de varios puntos entre las poblaciones indígenas con respecto al resto de la población (Bello y Rangel, 2002). En los casos más extremos, como Guatemala, México y Perú, la diferencia entre indígenas y no indígenas puede alcanzar de 20 a 30 puntos porcentuales (Psacharopoulos y Patrinos, 1994)1.

Efectivamente, el último informe sobre seguimiento mundial 2011 llamado "Mejorar las posibilidades de alcanzar los Objetivos del Milenio" del Banco Mundial y el Fondo Monetario Internacional, señala que uno de cada tres indígenas es pobre. Esta situación se ha visto afectada por el bajo crecimiento de la economía, junto con el limitado aporte que hacen los programas focalizados de reducción de la pobreza (BM, 2011).

Tabla 2

POBREZA INDÍGENA EN LATINOAMÉRICA

\begin{tabular}{|l|c|}
\hline País & Pobreza indígena \\
\hline México & 8 de cada 10 indígenas \\
\hline Ecuador & 7,8 de cada 10 indígenas \\
\hline Guatemala & 7,4 de cada 10 indígenas \\
\hline
\end{tabular}

Fuente: Banco Mundial y Fondo Monetario Internacional, 2011.

La conjugación de todos estos elementos (pobreza, marginación, carencias, etc.) ha desembocado en la "revitalización" de determinadas reivindicaciones y manifestaciones en defensa de su patrimonio, relacionadas especialmente con la lengua, la religión, la tradición cultural, las estructuras de organización socio-política, las dinámicas económicas, etc. (Hopenhayn y Bello, 2001).

En la década recién pasada aumentaron o se hicieron más visibles en el espacio público las demandas y reclamaciones de los pueblos indígenas, lo que podría sugerir que, a lo menos en algunos países de la región, ha emergido un nuevo actor social y, en algunos casos, un nuevo actor político que desafía a las formas tradicionales de hacer política ${ }^{2}$. Este hecho es relevante para el conjunto de las sociedades latinoamericanas

1. En Guatemala, México, Bolivia y Perú, el porcentaje de población indígena que vive en condiciones de pobreza supera el 60\% (Psacharopoulus y Patrinos, 1994; Hernández y Calcagno, 2003)

2. Entre los principales movimientos encontramos Unión de Naciones Indias (Brasil), Movimiento Tupaq Katari (Bolivia), Confederación de Nacionalidades Indias (Ecuador), Coordinación Regional de los Pueblos Indios de México y América Central, Asociación de los Indios de Colombia, Organización Nacional de los Indios de Colombia, Consejo de Todas las Tierras de los mapuches (Chile), Consejo Indio Sudamericano, Organizaciones Indias de la Cuenca del Amazonas y Parlamento Indígena de América. 
y para el futuro de la democracia, pues plantea el desafío de repensar la comunidad política y los mecanismos de inclusión ciudadana en que hasta ahora se han basado los modelos de sociedad (Bello, 2004).

Según algunos autores, en el último tiempo "el tema indígena tiene más presencia en el debate político, más reconocimiento en la opinión pública, más resonancia en los debates internacionales y más tradición en la investigación social, pues estos pueblos se encuentran más claramente diferenciados respecto del conjunto de la sociedad, ya que sus formas de organización los constituyen en actores sociales más definidos en el debate político y en la escena pública en general" (Hopenhayn y Bello, 2001).

Como señalan Hall y Patrinos (2004), en términos políticos "la influencia política de los pueblos indígenas de América Latina, medida según la cantidad de partidos políticos indígenas, representantes electos de origen indígena, disposiciones constitucionales a favor de los pueblos indígenas o políticas de salud y educación pertinentes, ha crecido de manera sorprendente durante los últimos 15 años. La democratización, la globalización y las presiones internacionales son razones que pueden explicar este notorio cambio" (Hall y Patrinos, 2004).

Por otra parte, a lo anterior hay que agregar que el nuevo contexto social, político y económico ha producido cambios en la estructura y presencia de los pueblos indígenas. La heterogeneidad y la diversidad de estos pueblos se expresa también hoy en los diferentes espacios geográficos que ocupan dentro de sus propios países. A pesar de que generalmente se los ha asociado con el medio rural, la migración campo-ciudad y el crecimiento natural de su población en zonas urbanas han configurado otra realidad (Psacharapoulos y Patrinos, 1994; Plant, 1998; Hopenhayn y Bello, 2001).

La migración indígena constituye uno de los fenómenos más importantes de los últimos tiempos, ya que a pesar de que muchos movimientos migratorios comenzaron en la época colonial, el mayor volumen y sus impactos se verifican principalmente desde mediados del siglo XX. Entre sus causas, Martín Hopenhayn y Álvaro Bello (2001) citan el deterioro de las economías campesinas, la pérdida y disminución de las tierras comunitarias, la carencia general de recursos productivos, el crecimiento de la población, la salarización y la pobreza, junto con otros factores subjetivos como la atracción por las "ventajas" de la vida en la ciudad.

Estos y otros elementos deberán ser considerados a la hora de analizar la especial situación que también viven los pueblos indígenas de Chile.

\section{LA REALIDAD DE LOS PUEBLOS INDÍGENAS EN CHILE: DIFERENTES PERSPECTIVAS PARA UN ANÁLISIS COMPLEJO}

La historia de los pueblos indígenas de Chile comparte algunas características comunes con otros pueblos indígenas latinoamericanos. En gran medida, la historia oficial de Chile muestra a la mayoría de estos pueblos como "guerreros, indómitos, valientes". En el caso del pueblo mapuche, estos se opusieron tenazmente a la conquista española, librando una guerra que duró más de 300 años y que culminó tardíamente a finales 
del siglo pasado. Los historiadores latinoamericanos, sin distinción, los señalan como "ejemplo de coraje y tenacidad para todos los pueblos de América" (Bengoa, 2000).

Sin embargo, actualmente estos pueblos están asociados a condiciones de pobreza y marginalidad. Durante los últimos años estas condiciones se han acentuado producto de las migraciones campo-ciudad y de medidas que poco o nada protegen sus derechos ancestrales. Esta situación los ha llevado a protagonizar movilizaciones y levantamientos orientados a reivindicar sus derechos y a mejorar su calidad de vida.

Una revisión de los principales datos asociados a estos pueblos nos permitirán conocer no solo su realidad actual, sino también tener una mirada retrospectiva que aporte mayores antecedentes para reflexionar en torno a sus demandas. Sin embargo, se debe tener en cuenta que la información que arrojan instrumentos de carácter cuantitativo constituyen solo una cara de la moneda. La resolución de los problemas que aquejan a los pueblos indígenas requiere de un análisis más complejo.

Por primera vez en Chile, en el Censo de Población y Vivienda del año 1992, se incorporó una pregunta para conocer el porcentaje de población indígena y extraer así conclusiones respecto de su situación particular. La pregunta utilizada era "¿Si Ud. es chileno, se considera perteneciente a alguna de las siguientes culturas?”, y solo daba opciones para identificarse con las tres etnias indígenas mayoritarias de Chile (mapuche, aymara o rapanui). De acuerdo a la información recogida, el 10,3\% de la población total del país mayor de 14 años se autoidentificó como perteneciente a algunos de estos tres grupos étnicos, lo que arrojó una cifra total de 998.385 indígenas: 504.986 hombres y 439.399 mujeres.

Dentro de este marco poblacional, la etnia mapuche era ampliamente mayoritaria dentro de la población indígena, con el 93\% del total, seguido de los aymara $(4,9 \%)$ y rapanui $(2,2 \%)$. En el último Censo de Población y Vivienda realizado en Chile en el año 2002, la pregunta para conocer el porcentaje de población indígena cambió. En esta ocasión se preguntaba: "¿Si Ud. es chileno, se declara perteneciente a alguna de las siguientes culturas?", extendiendo las opciones a las ocho etnias reconocidas en la legislación vigente de la época.

La modificación de la pregunta arrojó cambios significativos en los porcentajes encontrados, lo que dificulta en gran medida las posibilidades de hacer comparaciones objetivas. En este Censo de 2002, 692.192 personas, un 4,6\% de la población mayor de 14 años, se declaró como perteneciente a una de estas etnias, siendo 349.557 hombres y 342.635 mujeres.

$\mathrm{Al}$ respecto, cabe señalar que en el año 2006 se reconoció explícitamente la etnia diaguita, integrando, en el artículo $\mathrm{N}^{\circ} 1$ de la Ley 19.253, a este pueblo como parte de los pueblos indígenas que habitan en el territorio. Con ello, los nueve pueblos reconocidos en la ley son aymara, quechua, likan antai, colla, diaguita, rapanui, mapuche, kawéskar y yagán. Igual que en el censo anterior, la etnia mapuche es mayoritaria con un $87,3 \%$ de la población total, seguida de la aymara (7\%), atacameña (3\%). Sumando las restantes, un $2,7 \%$.

De acuerdo a la distribución de la población indígena por sexo, destaca que la proporción de hombres es levemente mayor a la de mujeres (50,5\% son hombres), situación 
inversa a la de la población no indígena del país. Sin embargo, desde una perspectiva de género y etnicidad, se puede sostener que hasta la fecha ha existido una invisibilización del impacto diferencial que la desigualdad social tiene sobre hombres y mujeres indígenas. Aun así, diversos estudios han demostrado que las mujeres indígenas son las más afectadas por las situaciones de pobreza, ya que sobre ellas recae el peso de una triple discriminación; por ser mujeres, pobres e indígenas (Peredo, 2004).

En un análisis comparativo entre pueblos se verifican situaciones especiales: en el pueblo aymara, la cantidad de hombres y mujeres es prácticamente la misma, mientras que en la rapanui, la cantidad de mujeres es mayor a la de los hombres. Esta tendencia puede apreciarse en la siguiente tabla:

Tabla 3

POBLACIÓN INDÍGENA POR SEXO

\begin{tabular}{|l|c|c|c|c|}
\hline \multirow{2}{*}{$\begin{array}{l}\text { Año/ } \\
\text { Sexo }\end{array}$} & \multicolumn{4}{|c|}{ Número } \\
\cline { 2 - 5 } & Mapuche & Aymara & Rapanui & Total \\
\hline 2002 & 604.349 & 48.501 & 4.647 & 657.497 \\
\hline Mujeres & 299.769 & 24.313 & 2.384 & 326.466 \\
\hline Hombres & 304.580 & 24.188 & 2.263 & 331.031 \\
\hline
\end{tabular}

Fuente: Censo 2002, INE

Respecto de la ubicación de la población indígena, cabe señalar que estos pueblos también han sufrido un proceso de desplazamiento a las ciudades, lo que no necesariamente ha significado mejoras en su calidad de vida, sino que se han visto enfrentados a un entorno muchas veces hostil que les ha obligado a vivir en condiciones marginales y de pobreza o de extrema pobreza (Peyser, 2003). Junto con ello, han tenido que adaptarse a un entorno cultural más complejo y muchas veces marcado por las pautas de la cultura dominante (Ancán, 1994; Bengoa, 1996).

La magnitud de esta migración indígena a las zonas urbanas ha cambiado los patrones con que antes se miraba este fenómeno, particularmente si se tiene en cuenta que en algunos países el balance de la población urbano/rural se inclina progresivamente hacia las ciudades. Es en este contexto donde observamos que casi el $70 \%$ de los pueblos indígenas habitan en zonas urbanas ocupando, como hemos dicho, zonas periféricas marginales, formando muchas veces núcleos de inmigrantes que dan continuidad a los nexos con sus tierras de origen (Psacharapoulos y Patrinos, 1994; Bello, 2004). Efectivamente, la comparación entre la Encuesta CASEN 2006 y la de 2009 muestra datos interesantes. En la del 2009, el 31,1\% de la población indígena vive en zonas rurales, porcentaje levemente superior al del año 2006, cuando alcanzaba a 30,6\%, pero muy inferior al de años anteriores. En 1996, el 48,3\% de la población indígena vivía en zonas rurales. 


\section{Tabla 4}

POBLACIÓN INDÍGENA POR ZONA DE RESIDENCIA

\begin{tabular}{|l|c|c|c|c|c|c|}
\hline \multirow{2}{*}{$\%$} & \multicolumn{2}{|c|}{2003} & \multicolumn{2}{c|}{2006} & \multicolumn{2}{c|}{2009} \\
\cline { 2 - 7 } & Indígena & No indígena & Indígena & No indígena & Indígena & No indígena \\
\hline Rural & 37,4 & 11,7 & 30,6 & 11,6 & 31,1 & 11,4 \\
\hline Urbano & 62,6 & 88,3 & 69,4 & 88,4 & 68,9 & 88,6 \\
\hline
\end{tabular}

Fuente: Elaboración propia sobre resultados CASEN 2003-2006-2009.

Respecto de su ubicación por regiones, la población indígena se concentra fundamentalmente en las regiones de La Araucanía (24,6\%), Metropolitana (24,0\%) y de Los Lagos (14,5\%). Sin embargo, en consideración al total de la población regional, la población indígena es proporcionalmente más importante en las regiones de La Araucanía $(30,1 \%)$, Arica y Parinacota (25,4\%), Magallanes y la Antártica Chilena (22,7\%), Aysén $(21,8 \%)$ y Los Lagos (20,8\%).

Sobre la situación de pobreza, esta ha aumentado durante los últimos años entre la población indígena. Efectivamente, en el año 2009 un 15,1\% de la población se encontraba en situación de pobreza, lo que equivalía a 2.564 .032 personas. Estas cifras contrastan con la situación de pobreza detectada en la CASEN del año 2006, la que afectaba al 13,7\% de la población del país. Ello significa un incremento de 1,4 puntos porcentuales, y que hay 355.095 pobres más que en 2006.

Similar comportamiento experimentó la indigencia o extrema pobreza. Mientras en 2006 el 3,2\% de la población se encontraba en esta situación, lo que equivale a 516.738 personas, en 2009 la indigencia se elevó hasta el 3,7\%, lo que representa 634.328 personas. Por zonas, se observa que, respecto del 2006, aumentó tanto la pobreza urbana como la rural, y se confirma la tendencia iniciada en 2006 de una mayor pobreza en zonas urbanas $(15,5 \%)$ respecto de zonas rurales (12,9\%) (Ministerio de Planificación, 2009).

Tabla 5

COMPARACIÓN DE POBREZA INDÍGENA / NO INDÍGENA

\begin{tabular}{|c|c|c|c|c|c|c|}
\hline \multirow{2}{*}{$\%$} & \multicolumn{2}{|c|}{2003} & \multicolumn{2}{c|}{2006} & \multicolumn{2}{c|}{2009} \\
\cline { 2 - 7 } & Indígena & No indígena & Indígena & No indígena & Indígena & No indígena \\
\hline Pobres & 29,4 & 18,1 & 19,0 & 13,3 & 19,9 & 14,8 \\
\hline
\end{tabular}

Fuente: Elaboración propia sobre resultados CASEN 2003-2006-2009.

Otro factor importante a destacar es la lengua y la cultura de los pueblos indígenas, donde los procesos de modernización acelerada han representado en todas partes un desafío y una amenaza para los mismos (Bengoa, 1996), aun cuando estos continúan presentes en la realidad chilena como culturas diferenciadas, adaptándose a los cambios e integrando aquellos aspectos de la cultura mayoritaria que les faciliten esta existencia. En ese sentido, algunos autores señalan que el proyecto que históricamente ha llevado 
a cabo el Estado chileno se tradujo en una fuerza histórica niveladora que eliminaba las diferencias y pluralidades encontradas para ponerlas bajo una única "cultura nacional", lo que involucró la exclusión de los indígenas por parte de la sociedad chilena (Millaleo, 1996).

Efectivamente, los datos muestran una tendencia hacia la desaparición de estas culturas, la que se está produciendo de forma acelerada durante el último tiempo. Por ejemplo, para el pueblo mapuche, un estudio realizado en 1996 (Millaleo, 1996) afirma que se encuentran fuertes procesos de reforzamiento de la identidad indígena: las personas señalaban con más fuerza su pertenencia al pueblo mapuche, se hablaba más el mapudungún, se realizaban más ceremonias y festividades propias de la cultura mapuche.

El año 2009 solo el 12,0\% de la población indígena hablaba y entendía su lengua originaria y el 10,6\% solo entendía. Como contrapartida, el 77,3\% de la población indígena no habla ni entiende su lengua originaria, cifra que es 5,6 puntos porcentuales mayor que la de 2006, cuando alcanzaba a $71,7 \%$. Son los adultos y adultos mayores quienes más hablan y/o entienden su lengua originaria, mientras que las generaciones más jóvenes la han ido perdiendo. El 42,3\% de la población indígena adulto mayor habla y/o entiende su lengua originaria, mientras que la cifra alcanza a solo 10,6\% en el caso de la población indígena de entre 0 y 17 años ${ }^{3}$.

Lo anterior adquiere suma relevancia, ya que, tal como señala el Informe de la UNICEF 2001, "el uso de las lenguas es un elemento central en la definición de la cultura e identidad de los pueblos. Su conocimiento y uso por parte de la población permite visualizar procesos de transmisión y preservación cultural, así como también la identificación de fenómenos que inciden en la pérdida y fortalecimiento de la identidad de los pueblos originarios" (UNICEF, 2011: 23)

De acuerdo con la Encuesta CASEN 2006 y 2009, los datos arrojaban las siguientes cifras:

Tabla 6

POBLACIÓN INDÍGENA QUE HABLA / ENTIENDE SU LENGUA

\begin{tabular}{|l|c|c|}
\hline Pueblo/año & 2006 & 2009 \\
\hline Habla y entiende & 14,2 & 12,0 \\
\hline Solo entiende & 14,1 & 10,6 \\
\hline No habla ni entiende & 71,7 & 77,3 \\
\hline
\end{tabular}

Fuente: Elaboración propia sobre resultados CASEN 2006-2009.

Estas cifras adquieren una nueva dimensión cuando se consideran a los niños y jóvenes indígenas. Como señala la UNICEF, desde el año 2000 se observa una pérdida de la utilización de las lenguas originarias en la población mayor y menor de 18 años de edad. El año 2009, el 89,4\% de la población infantil indígena declara no hablar ni

3. Véase: http://www.ministeriodesarrollosocial.gob.cl/casen2009/indigena.php 
entender ninguna lengua originaria, un 6,9\% solo entiende y un 3,7\% habla y entiende. Entre los años 2000 y 2009, disminuyeron en un 37,3\% los menores de edad que solo entienden la lengua. El análisis desagregado sobre el uso y comprensión de las lenguas originarias, según rangos etarios y zona de residencia, devela una brecha generacional. En este sentido, la población sobre 45 años habla y entiende en mayor proporción la lengua (UNICEF, 2011).

En la zona urbana, el 10,2\% de la población entre 46 y 59 años habla y entiende alguna lengua, en tanto solo un 2,4\% de los adolescentes entre 14 y 17 años lo hace. En la zona rural se observa una mayor utilización de las lenguas originarias que en la zona urbana en todos los grupos de edad. No obstante, la brecha generacional se mantiene; el 33,5\% de la población entre 46 y 59 años habla y entiende, y un $11 \%$ de los adolescentes entre 14 y 17 años lo hace (UNICEF, 2011).

Finalmente, nos parece relevante tomar otra dimensión de la problemática indígena que tiene que ver con sus indicadores de salud. Si bien no existen datos desagregados de acuerdo a cifras oficiales, resulta interesante recoger algunos datos que arrojó un estudio llevado a cabo en el año 2009 a partir del proyecto de "epidemiología con enfoque sociocultural" del Ministerio de Salud, el que busca generar un panorama nacional sobre la salud de los pueblos indígenas de Chile a partir de información disponible y con una metodología innovadora, en el marco de los determinantes sociales y con énfasis en los derechos colectivos de los pueblos (Oyarce y Pedrero, 2009).

Hasta el momento se han generado indicadores para el pueblo aymara de las regiones de Tarapacá y Arica y Parinacota; para el pueblo mapuche residente en la Región de Los Ríos, la Provincia de Arauco (Región del Bío-Bío) y las comunas del área lafkenche de la Provincia de Cautín (Toltén, Teodoro Schmidt, Carahue y Saavedra) en la Región de La Araucanía; y para los pueblos yámana y kawéskar del extremo sur de Chile en la Región de Magallanes, donde se analizó, además, la situación de los mapuche-williches migrantes que residen en ese territorio. Si bien se trata de una información limitada, permite evidenciar algunas realidades propias para estos pueblos, que definirían la necesidad de una intervención diferenciada.

Este estudio muestra que las tasas brutas de mortalidad calculadas para el trienio 2001-2003 muestran sistemáticamente la sobremortalidad de las poblaciones indígenas, cuyos mayores riesgos de morir fluctúan entre un 30\% para los mapuches del área lafkenche de La Araucanía (riesgo relativo de 1,3) y un $80 \%$ para los indígenas del extremo sur (riesgo relativo de 1,8) (Oyarce y Pedrero, 2009).

En ese sentido, las autoras señalan que "la sobremortalidad indígena es un rasgo común en todos los grupos de edad, sin distinciones de sexo ni área de residencia para todos los pueblos estudiados, lo que estaría indicando que uno de los determinantes estructurales de este perfil es la etnia, que actuaría como un estratificador social por medio de la discriminación. Es decir, no son los modos de vida indígenas los que condicionan el mayor daño, sino la relación étnica con los no indígenas o, dicho de otro modo, la posición subordinada de estos pueblos en la estructura social del país" (Oyarce y Pedrero, 2009: 132). 


\section{ALGUNAS REFLEXIONES FINALES: EVIDENCIAS Y DESAFÍOS PARA LAS POLÍTICAS PÚBLICAS INDÍGENAS}

La información que arrojan diferentes instrumentos agregados, como el Censo o las Encuestas de Caracterización Socioeconómica, constituyen fuentes fundamentales para la toma de decisiones, al entregarnos evidencias no solo de una realidad particular, sino también al permitir evidenciar el resultado de diferentes políticas públicas a lo largo del tiempo.

Sin embargo, más allá de estas aportaciones, será necesario comprender que difícilmente se podrá mejorar la situación de los pueblos indígenas si solo se considera esta información. Entre los principales aportes que arroja una caracterización demográfica, está la visibilización física de esta población, cuantificar sus principales problemas y necesidades, identificar sus rangos etáreos, reconocer las temáticas sanitarias que los aquejan, etc. Pero sin duda lo fundamental será cómo se "problematizan" estas situaciones y cómo se definen sus soluciones, las que no pasan por una sola mirada disciplinar o especializada, sino más bien en el mix que se puede hacer de todas ellas.

Es aquí donde adquiere relevancia la participación de los pueblos indígenas en la toma de decisiones que les atañen, al ser ellos los principales actores afectados y que pueden aportar soluciones desde su propia cosmovisión, sin perder la dimensión cultural que subyace a sus problemáticas. Esto porque son varias las situaciones complicadas que deben enfrentar los pueblos indígenas, donde la diversidad y la multiculturalidad de las sociedades actuales no han implicado, por lo general, respeto ni un mayor nivel de pluralismo o integración cultural (Etxeberria, 2004).

Por una parte, esta negación de la heterogeneidad cultural que ha caracterizado el nacimiento de la mayoría de los Estados nacionales se ha perpetuado en el tiempo mediante sistemas de reproducción social y cultural (Hopenhayn y Bello, 2001). Han existido diversas formas mediante las cuales los pueblos indígenas se han incorporado a las comunidades políticas: desde la conquista y la colonización de sociedades que anteriormente gozaban de autogobierno hasta la inmigración voluntaria de individuos y familias (Kymlicka, 1996).

Tal como señala Kymlicka, por regla general los pueblos indígenas han estado aislados del proceso de formación estatal y de aquellos que afectaban su propio desarrollo (Kymlicka, 2003). Este proceso ha significado para muchos de los indígenas la pérdida progresiva de tierras, el quiebre de economías comunitarias, la pérdida de derechos y representatividad y la marginación de procesos políticos, entre otros.

En el caso chileno, los datos presentados parecen demostrar no solo la urgencia de asumir una visión diferenciada a la hora de abordar la problemática situación de los pueblos indígenas, sino también de entender que este proceso se debe hacer de manera participativa. Si bien no basta con recoger una serie de datos sociodemográficos para dar cuenta de la realidad de pobreza, pérdida cultural, creciente urbanidad, falta de oportunidades, entre otros, sí consideramos que su análisis se convierte en una fuente para proponer nuevas alternativas y visiones. 
La emergencia étnica es, en este sentido, el reflejo de una incipiente conciencia ciudadana que busca cauces de participación y nichos de inclusión desde los parámetros de la diversidad cultural y la identidad. Lo anterior requiere encontrar nuevos modos de conjugar la dimensión universal de la ciudadanía y la sociedad civil con las diferencias específicas de estos pueblos (Hopenhayn y Bello, 2001).

Es por ello que existe la necesidad de explorar nuevas vías que permitan dar respuesta a las necesidades planteadas y que permitan ejercer una ciudadanía efectiva de estos pueblos, entendida desde la diversidad. Este ejercicio no solo requiere la apertura de vías de participación, sino la adecuación de los mecanismos de decisión que les permitan ejercer una influencia directa sobre sus alternativas de desarrollo, transfiriendo a los pueblos indígenas cuotas de poder para definir sus propias vías de acción y participación, es decir, su empoderamiento (Durston, 2002).

Sin embargo, los factores culturales que subyacen a estos pueblos, y que podrían enriquecer el proceso de formulación de políticas públicas, no siempre son considerados. De hecho, en algunos estudios, las principales críticas a las políticas públicas dirigidas a los pueblos indígenas se relacionan con la ineficiencia de las intervenciones, el sesgo en lo que se considera relevante, la falta de espacios de participación efectivos, entre otros (Fonseca, 2001; Peyser, 2003; Hall y Patrinos, 2004).

Alkire (2005), en un estudio sobre pobreza indígena e intervención externa, señala que las principales críticas a las políticas públicas son las siguientes: a) Definición sesgada de programas y proyectos, pues al no entender adecuadamente la influencia cultural de un pueblo se asumen posturas erróneas acerca de determinadas conductas o valores; b) Imposición de valores, pues se intenta deliberadamente cambiar normas culturales o prácticas que se consideran negativas pero que no son entendidas en contextos culturales diversos; c) Recursos sin potenciar, especialmente por no tomar en cuenta métodos de trabajo, formas de relación, riqueza cultural, etc.; d) Imprevisión de impactos no deseados, pues no han entendido o no han anticipado factores negativos para ellos.

Otro problema que se menciona es que la mayoría de las políticas públicas indígenas enfatizan demasiados aspectos cuantitativos, materiales o económicos, dejando a un lado aspectos sociales y culturales por considerarlos de menor importancia, pero que claramente forman parte de las demandas de los pueblos indígenas. En ese sentido, los estudios identifican fallas de dos tipos: a) no se han recogido adecuadamente las prioridades de estos pueblos, y b) ha existido un pobre entendimiento de las relaciones entre componentes sociales, culturales, económicos, políticos y ecológicos propios de estos pueblos (Pirttijärvi, 1999; Rey, 2002).

Todo lo anterior indica que los pueblos indígenas no pueden ser entendidos y atendidos dentro de "categorías mayores", como ha sido la tónica de muchas iniciativas (pobres, campesinos, desempleados, etc.), sino que se necesita de un análisis mucho más profundo, más focalizado pero, a su vez, más integrado y complejo, de manera de rescatar y considerar sus propias especificidades culturales. Esto impone nuevos desafíos a la identificación de oportunidades para el desarrollo de políticas públicas que incorporen a los pueblos indígenas. 
Para la mayoría de los pueblos indígenas latinoamericanos, la realización de su plan de vida depende de la existencia y de la preservación, o incluso del florecimiento y el desarrollo de su cultura. En una sociedad multicultural como la actual, los Estados deben ser capaces de crear las condiciones para garantizar la satisfacción de las necesidades (en su sentido más amplio) de todas las personas (Olivé, 2004). Esto requerirá de la existencia de espacios públicos culturalmente inclusivos y socialmente diversos. Sin embargo, como hemos venido señalando a lo largo de esta investigación, la exclusión de las culturas originarias de la cultura política no ha permitido la formación de una cultura ciudadana pluralista e interculturada, necesaria en el mundo en el que vivimos (Tubino, 2004).

Para apoyar la formulación de políticas públicas pertinentes será necesario vincular algunos de los datos sociodemográficos presentados con otro tipo de información relevante. Será necesario, por tanto, conocer qué tipo de relaciones establecen con su entorno próximo y lejano, el significado que atribuyen a los vínculos sociales con sus pares y otros actores sociales, particularmente en el espacio local, cómo se fortalece la cultura y la lengua, cuáles son los pilares que sustentan la memoria histórica de estos pueblos, aun cuando se encuentran en zonas urbanas. Esta dimensión es de suma relevancia cuando visualizamos a estos pueblos como actores protagónicos en la superación o mejora de sus condiciones de vida.

Esto es fundamental para que las políticas públicas contribuyan a un verdadero desarrollo con identidad, al nutrirse de la mirada particular de los pueblos indígenas, ya que "la modernización ha logrado generar mejores niveles de igualdad social y de libertad política en aquellas sociedades donde se gestó a partir de tradiciones propias. El problema surge cuando se moderniza arrasando las tradiciones locales y no arraigándose en ellas" (Tubino, 2004).

Por lo tanto, aquellas deben permitir la participación de los pueblos indígenas de acuerdo a sus propios patrones culturales, definirán la naturaleza de sus recursos, sus criterios de membresía y la forma en la que se estructuraran sus redes, siendo poseedores de un capital social propio que opera de acuerdo a normas y valores básicos, con mecanismos propios de aplicación de sanciones y con formas establecidas para compartir el conocimiento y la información disponible (Durston, 1999; Robinson y Williams, 2004).

Como señala Bernardo Kliksberg (2000), "el capital social y la cultura son componentes claves de las interacciones de una comunidad. Las comunidades son portadores de actitudes de cooperación, valores, tradiciones, visiones de la realidad, que son su identidad misma. Si ello es ignorado, salteado, deteriorado, se inutilizarán importantes capacidades aplicables al desarrollo, y se desatarán poderosas resistencias. Si, por el contrario, se reconoce, explora, valora, y potencia su aporte, puede ser muy relevante y propiciar círculos virtuosos con las otras dimensiones del desarrollo" (Kliksberg, 2000). El desafío será, por tanto, considerar en la toma de decisiones aquellas fuentes de información que nos permiten caracterizar a los pueblos indígenas, sin perder de vista la multidimensionalidad que caracteriza su realidad. 


\section{REFERENCIAS BIBLIOGRÁFICAS}

Alkire, S., "Subjective Quantitative Studies of Human Agency", en Social Indicators Research, Vol. 74, $\mathrm{N}^{\circ} 1,2005$, págs. 211-217.

Ancán, J., "Los urbanos: un nuevo sector dentro de la sociedad mapuche contemporánea", en Pentukun, $\mathrm{N}^{\circ} 1,1994$, págs. 5-15.

Banco Mundial, Mejorar las posibilidades de alcanzar los Objetivos del Milenio, Washington: BM, 2011.

Bello, A., "Etnicidad y ciudadanía en América Latina: la acción colectiva de los pueblos indígenas", en Serie Políticas Sociales, № 79, Santiago: CEPAL, 2004.

Bello, A., Rangel, M., "La equidad y la exclusión de los pueblos indígenas y afro descendientes en América Latina y el Caribe" en Revista CEPAL, N 76, abril 2002.

Bengoa, J., "Población, familia y migración mapuche. Los impactos de la modernización en la sociedad mapuche 1982-1995", en Revista Pentukun, № 6, 1996, págs. 9-28.

— Historia del pueblo mapuche. Siglo XIX y XX, Santiago de Chile: LOM Ediciones, 2000.

Deruyttere, A., "Nativos en números", en Revista Bidamérica, septiembre-octubre 1999, 1999, pág. 15.

Domenech, E., "El multiculturalismo en Argentina: Ausencias, ambigüedades y acusaciones", en Revista Estudios, № 14, 2003, págs. 33-47.

Durston, J., Análisis del aporte del capital social campesino a la superación de la pobreza rural, Informe descriptivo final, CEPAL, División de Desarrollo Social, 1999.

- "Capital social: parte del problema, parte de la solución, su papel en la persistencia y en la superación de la pobreza en América Latina y El Caribe", en CEPAL (eds.), Capital social y reducción de la pobreza en América Latina y El Caribe: en busca de un nuevo paradigma, CEPAL y Michigan State University, 2003.

Etxeberria, X., Sociedades multiculturales, Bilbao: Alboan/Mensajero, 2004.

Fonseca, R., "El trabajo y la inclusión social desde la perspectiva del desarrollo", véase online en http://www.iigov.org/dhial/?p=7_08, (15/08/2010).

Hall, G., Patrinos, A., Pueblos Indígenas, pobreza y desarrollo humano en América Latina: 1994-2004, Informe Banco Mundial, 2004.

Hernández, I., Calcagno, S., Los pueblos indígenas y la sociedad de la información en América Latina y el Caribe: un marco para la acción, véase online en http://www.mapuche.info/mapuint/hernacalca030400s.pdf, (02/05/2012).

Hopenhayn, M., "iIntegrarse o subordinarse? Nuevos cruces entre política y cultura", en Mato, D., (ed.), Estudios latinoamericanos sobre cultura y transformaciones sociales en tiempos de globalización, Buenos Aires: CLACSO, 2001.

Hopenhayn, M., Bello, A., "Discriminación étnico-racial y xenofobia en América Latina y El Caribe", en Serie Políticas Sociales, No 47, Santiago: CEPAL, 2001.

Kliksberg, B., "Capital social y cultura, claves olvidadas del desarrollo", en Documento de divulgación, No 7, Banco Interamericano de Desarrollo (BID), 2000.

Kymlicka, W., La politica vernácula. Nacionalismo, multiculturalismo y ciudadanía, Buenos Aires: Piados, 2003.

Máiz, R., "El indigenismo político en América Latina", en Revista de Estudios Políticos (Nueva Época), No 123, enero-marzo 2004, págs. 129-174.

Millaleo, S., Ser o no ser mapuche o mestizo, véase online en http://www.soc.uu.se/mapuche/mapuint/millaleo021123.html, (08/08/2010) 
Olivé, L., "Relaciones interculturales y justicia social: una fundamentación pluralista", en CastroLucic, M. (ed.), Los desafíos de la interculturalidad: Identidad, política y derecho, Vicerrectoría de Investigación y Desarrollo, Universidad de Chile, 2004.

Oyarce, A., Pedrero, M., "Una metodología innovadora para la caracterización de la situación de salud de las poblaciones indígenas de Chile: limitaciones y potencialidades", en Notas de población, No 89, CEPAL, 2009

Pedrero, E., Una aproximación a la problemática de género y etnicidad en América Latina, Santiago de Chile: CEPAL, 2004.

Peyser, A., Desarrollo, cultura e identidad: El caso del mapuche urbano en Chile. Elementos y estrategias identitarias en el discurso indígena urbano, Ph.D. Thesis, Universidad Católica de Louvain, 2003.

Pirttijärvi, J., Indigenous Peoples and Development in Latin America, University of Helsinki, Finlandia, 1999.

Plant, R., "Pobreza y desarrollo indígena: Algunas reflexiones", en Documento BID, No IND-105, 1998.

Psacharopoulos, G., Patrinos, H., Indigenous People and Poverty in Latin America: An Empirical Analysis, Washington: World Bank, Regional and Sectorial Studies, 1994.

Rey, G., "Cultura y desarrollo humano: unas relaciones que se trasladan", en Revista Digital de Cultura Pensar Iberoamérica, véase online en http://www.campus-oei.org/pensariberoamerica/ric00a04.htm, 2002, (23/10/2010).

Robinson, D., Williams, T., "Social Capital and Voluntary Activity: Giving and Sharing in Maori and non-Maori Society”, en Social Policy Journal of New Zealand, Nº17, 2004, págs. 52-71.

Touraine, A., "¿Qué es una sociedad multicultural? Falsos y verdaderos problemas", en Revista Claves de razón Práctica, N 56, 1995, págs. 14-25.

Tubino. F., "La impostergable alteridad: del conflicto a la convivencia intercultural", en CastroLucic, M. (ed.) Los desafíos de la interculturalidad: Identidad, política y derecho, Vicerrectoría de Investigación y Desarrollo, Universidad de Chile, 2004.

UNICEF, Incluir, sumar y escuchar: Infancia y Adolescencia Indígena, Santiago de Chile, 2011.

Valdés, M., El problema de lo urbano y lo rural, véase online en http://www.mapuche.cl/documentos, 2000, (11/11/2010).

Vallescar Palanca, D., Cultura, multiculturalismo e interculturalidad: hacia una racionalidad intercultural, Madrid: PS, 2000. 